Niclas Bockelmann, Jan Graßhoff, Lasse Hansen, Giacomo Bellani, Mattias P. Heinrich, and Philipp Rostalski

\title{
Deep Learning for Prediction of Diaphragm Activity from the Surface Electromyogram
}

https://doi.org/10.1515/cdbme-2019-0005

\begin{abstract}
The electrical activity of the diaphragm (EAdi) is a novel monitoring parameter for patients under assisted ventilation and is used for assessing the patient's neural respiratory drive. It is recorded by an array of electrodes placed inside the esophagus at the level of the diaphragm. A noninvasive alternative is the measurement of the electromyogram by means of skin surface electrodes (sEMG). The respiratory sEMG signal, however, is subject to electrocardiographic interference and crosstalk from other muscles and may also pick up a different part of the muscular activity. In this work, we propose to use a deep neural network to predict the electrical activity of the diaphragm as measured by a nasogastric catheter from sEMG measurements. We use a ResNet based architecture and train the network to directly regress the EAdi as a supervised learning task - we further investigate a heatmap based regression approach. The proposed methods are evaluated on a clinical dataset consisting of 77 recordings from mechanically ventilated patients. For the direct regression task, the network's predictions reach a Pearson correlation coefficient (PCC) of 0.818 with EAdi on the hold-out set. The heatmap regression increases the PCC to 0.830 while at the same time achieving a lower mean absolute error, indicating a superior performance. From our results we conclude that sEMG measurements may be used to predict the internal activity of the diaphragm as measured invasively using a nasogastric catheter.
\end{abstract}

Keywords: diaphragm activity, sEMG, deep learning

\section{Introduction}

In the light of recent studies regarding the prevalence and consequence of patient-ventilator asynchrony (PVA), researchers have emphasized the importance of monitoring the respiratory activity of patients under assisted spontaneous ventilation. In

\footnotetext{
Niclas Bockelmann, Jan Graßhoff, Philipp Rostalski, Institute for Electrical Engineering in Medicine, Universität zu Lübeck niclas.bockelmannestudent.uni-luebeck. de Lasse Hansen, Mattias P. Heinrich, Institute of Medical Informatics, Universität zu Lübeck hansen@imi.uni-luebeck. de

Giacomo Bellani, Università degli Studi Milano Bicocca, Dipartimento di Medicina e Chirurgia, Milano, Italy giacomo.bellanil@unimib.it
}

particular it was shown that PVA is associated with adverse outcomes such as a higher mortality and a prolonged duration of mechanical ventilation $[2,12]$. The electrical activity of the diaphragm (EAdi) was introduced as a novel monitoring parameter for the assessment of diaphragmatic function. It represents the envelope of the crural diaphragm's electromyogram (EMG), measured by a special nasogastric catheter equipped with an array of electrodes placed inside the esophagus. It was suggested that the amplitude of EAdi directly reflects the breath-to-breath neural output of the respiratory center [5]. In daily clinical practice, the insertion and proper positioning of the esophageal catheter can be cumbersome, which limits the routine application of EAdi. A non-invasive alternative is the skin surface electromyogram (sEMG), measured on the patients thorax. Despite enabling a direct clinical application, the respiratory sEMG comes with its own intricacies: the signalto-noise ratio is small and the measured signal is subject to strong electrocardiographic interference and muscle crosstalk. Also the electrodes are located at a different spatial position in comparison to the internally placed electrodes. Some researchers have considered the sEMG as a measure for patient activity [5] but it is still a subject of ongoing research to what extent it is a viable surrogate for EAdi. In [1] the sEMG data were processed by detecting and gating out cardiac artifacts, then the amplitude of the sEMG's envelope was compared to EAdi. The authors found a good correlation between EAdi and one of the sEMG channels when averaging over several breaths. In this work, we consider the problem of predicting the EAdi signal on a sample-by-sample basis from all sEMG channels. We use a deep neural network to learn the relation between the measured sEMG signals and the internal diaphragm activity. We train a ResNet based architecture and, given the high noise level of the input signals, we further investigate a heatmap regression approach.

\section{Material and Methods}

\subsection{Data}

The data consist of 77 clinical recordings from a total of 23 patients receiving pressure support ventilation, with recording lengths varying between 2 and 15 minutes. The data were 
recorded in an 8-bed ICU at the San Gerardo Hospital (Monza, Italy), see [1] for further details on the data collection. Each dataset contains four raw sEMG channels and EAdi as the regression target, respectively sampled at $500 \mathrm{~Hz}$ and $100 \mathrm{~Hz}$. The sEMG channels were recorded with pairs of electrodes positioned (1) bilaterally at the lower costal margin on the midclavicular line ("costmar"), (2) bilaterally in the second intercostal space ("intercost"), (3) parallel to the muscle fibers above the rectus abdominis muscle ("rect"), (4) parallel to the muscle fibers above the sternocleidomastoid muscle ("sterno") and (5) bilaterally $5 \mathrm{~cm}$ below the costmar-electrodes ("belowcost"). The first three channels are included in all recordings, while from the last two channels only one is available in each patient - the respective other channel was set to zero prior to training. An exemplary segment of the raw sEMG data is depicted in the top part of Figure 1.

\subsection{Architecture}

For the proposed supervised learning task we use a deep convolutional neural network (DCNN) with 34 layers (cf. Figure 1). The number of layers of the DCNN was empirically determined to yield a sufficient receptive field and degree of semantic abstraction considering the challenging problem of predicting diaphragm activity from raw sEMG signals. To ease the training of the $\mathrm{CNN}$ we make use of residual blocks by incorporating identity skip connections [6] between convolutional layers. Features are downsampled after every third residual block using strided convolutions. At the same time we double the number of feature channels to enrich semantic information. Motivated by [3], a kernel size of seven is used in all residual blocks, whereas in the first layer of the network a large kernel of size 51 is employed to enlarge the receptive field and capture and process low level signal features. Following the residual blocks, upsampling and convolution operators are used to propagate semantic features to higher resolution layers. A final convolutional layer with kernel size seven maps the features to the number of output channels. Rectified Linear Units (ReLUs) are used throughout the network as non-linear activation functions. For faster training convergence and improved generalization we employ batch normalization [7].

\subsection{Training}

Input to the DCNN are the previously described sEMG signals (costmar, intercost, rect, belowcost and sterno). To deal with the missing channels in the recordings (belowcost or sterno) we set the respective input channel to zero. The network is trained with fixed length patches of size 5120 (corresponding

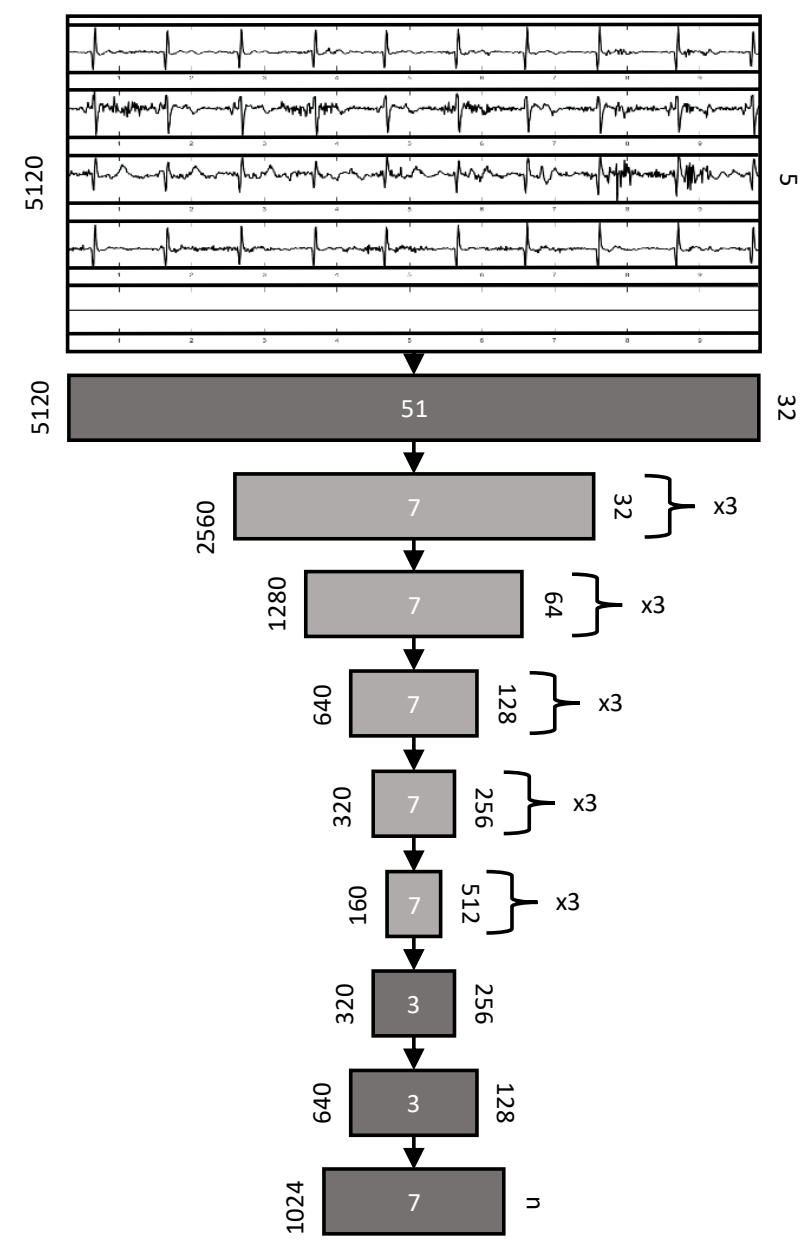

Fig. 1: Overview of the proposed network architecture. Light gray boxes represent residual blocks containing two convolution layers and a skip connection. Dark gray boxes represent upsampling modules containing an upsampling operator and additional convolutional layers. Numbers denote the kernel size (on box), signal size (left of box) and the number of feature channels (right of box) The numbers next to the curly bracket indicate repetition of residual blocks. Convolutional layers are followed by batch normalization and non-linear activations.

to time intervals of approx. 10 seconds), that are randomly cropped from the input data. The corresponding EAdi signal of size 1024 serves as the regression target. Prior to training, sEMG signals are normalized to have standard deviation of one. One of the challenges in predicting the EAdi is that it may be arbitrarily scaled against other EMG channels due to differences in the electrode/tissue impedance [1]. These scaling factors between the EMG modalities can not be predicted from the data itself. Therefore, prior to training, we normalize the EAdi amplitude to either the intercost or the costmar envelope (depending on which of the two has a higher correlation to EAdi), and thereby remove the scaling ambiguity. We train the proposed DCNN architecture with two different regression tasks: (1) direct regression of the EAdi signal (output channel 
Tab. 1: Results of different methods on the hold-out set.

\begin{tabular}{lccc}
\hline Method & PCC & MAE & HS \\
\hline DirectNet & $0.818 \pm 0.198$ & $1.071 \pm 0.835$ & $0.856 \pm 0.064$ \\
HeatNet & $\mathbf{0 . 8 3 0} \pm 0.193$ & $\mathbf{0 . 8 3 5} \pm 0.700$ & $\mathbf{0 . 8 8 8} \pm 0.044$ \\
Envelope & $0.799 \pm 0.197$ & $1.219 \pm 0.932$ & $0.765 \pm 0.066$ \\
\hline
\end{tabular}

Tab. 2: Results of the trained networks on the hold-out set for only using a single input channel while other channels are set to zero.

\begin{tabular}{llccc}
\hline Method & Channel & PCC & MAE & HS \\
\hline DirectNet & Costmar & $0.787 \pm 0.224$ & $1.400 \pm 1.245$ & $0.828 \pm 0.074$ \\
DirectNet & Intercostn & $0.176 \pm 0.269$ & $1.560 \pm 1.158$ & $0.711 \pm 0.032$ \\
DirectNet & Rect & $-0.003 \pm 0.196$ & $1.570 \pm 1.166$ & $0.707 \pm 0.031$ \\
DirectNet & Belowcost & $-0.035 \pm 0.220$ & $1.570 \pm 1.163$ & $0.706 \pm 0.032$ \\
DirectNet & Sterno & $0.020 \pm 0.207$ & $1.566 \pm 1.159$ & $0.707 \pm 0.031$ \\
\hline HeatNet & Costmar & $0.812 \pm 0.208$ & $0.895 \pm 0.719$ & $0.860 \pm 0.066$ \\
HeatNet & Intercostn & $0.000 \pm 0.208$ & $1.576 \pm 1.163$ & $0.707 \pm 0.031$ \\
HeatNet & Rect & $-0.003 \pm 0.202$ & $1.578 \pm 1.164$ & $0.703 \pm 0.035$ \\
HeatNet & Belowcost & $-0.042 \pm 0.241$ & $1.581 \pm 1.163$ & $0.699 \pm 0.031$ \\
HeatNet & Sterno & $-0.001 \pm 0.212$ & $1.574 \pm 1.158$ & $0.705 \pm 0.031$ \\
\hline
\end{tabular}

$n=1$ ) and (2) heatmap regression (output channel $n=128$ ), where the regression target is a quantized Gaussian distribution with a standard deviation $\sigma=2 \mu \mathrm{V}$ and the mean taken from the EAdi signal. To obtain the final single channel signal we take a Gaussian smoothed argmax from the quantized heatmap. Regressing a heatmap target rather than single values has been proven advantageous in terms of robustness and convergence of training in many different deep learning tasks $[9,11]$. We implement our regression framework in PyTorch [10]. All models are trained for 650 epochs with a batch size of 26 using Adam optimization [8] with an initial learning rate of 0.01. L1 and L2 loss is used for the direct and heatmap regression, respectively.

\subsection{Experiments}

The recordings of the 77 patients are split into a training and test set with 52 and 25 recordings, respectively. To evaluate the performance of the regressed predictions we report results for three different metrics: the Pearson correlation coefficient (PCC), the mean absolute error (MAE) and the Hamming similarity (HS). The reference for the evaluation is again the EAdi signal. The Hamming similarity was calculated as a metric of how well the derived signals can detect the timing of inspirations and expirations compared to EAdi. To this end, we used a simple breath detector (based on an adaptive thresholding algorithm similar to [4]) and calculated the Hamming distance of the detected inspiratory/expiratory samples to the inspiratory/expiratory samples detected in EAdi. All results are obtained from the recordings of the hold-out set and we refer to the direct regression and the heatmap regression as DirectNet and HeatNet, respectively. As a further baseline we evaluate the same performance metrics on the conventional envelope of the costmar sEMG channel (calculated by gating out cardiac artifacts first and then using a running root-mean-square filter as described in [1]). In an additional experiment we investigate the relevance of the different input signals for the regression task. Therefore, we evaluate our trained networks using only a single channel as the input while setting the remaining channels to zero and report the corresponding evaluation metrics for each sEMG channel separately. Lastly, we report the inference times of our regression model: One sEMG signal patch of $10 \mathrm{~s}$ is processed in approximately $6 \mathrm{~ms}$ on an NVIDIA RTX $2070 \mathrm{GPU}$ and $40 \mathrm{~ms}$ on an INTEL Core i7-6700K CPU.

\section{Results and Discussion}

The quantitative results of the different sEMG-based predictors are provided in Table 1. For the prediction of diaphragm activity, both neural network approaches clearly outperform the simple costmar envelope function, that has been used in previous publications (e.g. [1]). In comparison to the direct regression, the application of a heatmap regression procedure showed an improvement by $1.5 \%$ in PCC and by $22.0 \%$ in MAE, while the HS is improved by 3.2 percentage points. These results indicate a superior performance of the heatmap regression over the direct regression for our given task. The performance metrics for evaluating the neural networks on a single sEMG channel are given in Table 2. It turns out, that for both architectures, the costmar channel is the best predictor for EAdi. Still, we achieve the best performance, when using all sEMG channels at once, which might indicate, that all of the considered electrode positions on the thorax provide some information on the internal diaphragm activity. An exemplary segment from a patient in the hold-out set is depicted in Figure 2 - in that segment the sEMG data had a particularly low signal-to-noise ratio, which is reflected by the substantial noise visible in the costmar envelope. Both neural network methods nicely capture the internal diaphragm activity. Visually, the HeatNet appears to better match the on-off characteristic of the EAdi signal when comparing it to the direct regression approach (see seconds 12 to 15 ). Both neural networks have a high sensitivity to crosstalk artifacts in the sEMG signals (e.g. motion artifacts), which is exemplified in Figure 3. In that segment, both the HeatNet regression and the sEMG envelope show large artifacts, that do not correspond to internal diaphragm activity. This problem will be addressed in further studies. 


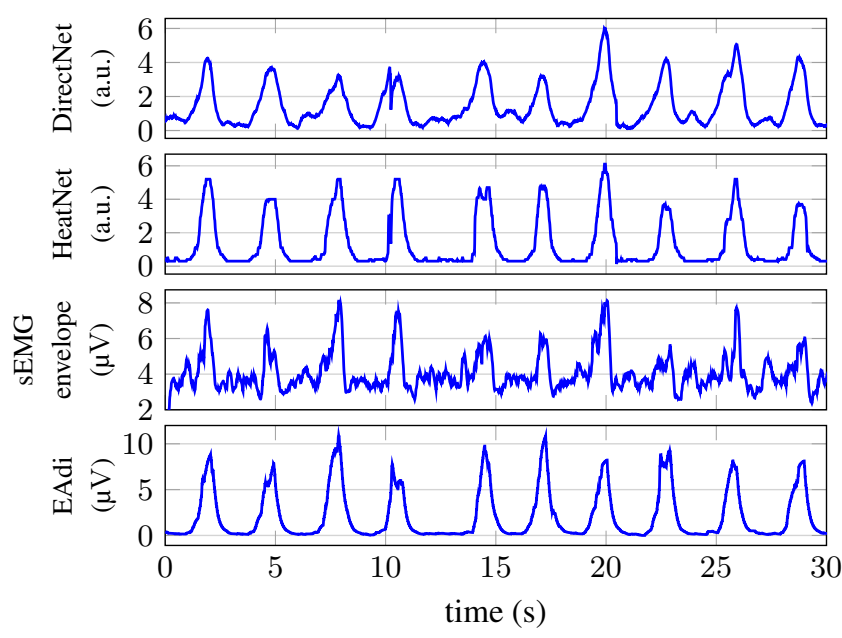

Fig. 2: Exemplary result on a patient from the hold-out set. The DirectNet and HeatNet results are shown for three concatenated patches (each approx. ten seconds long). The conventional sEMG envelope (costmar channel) and EAdi are given as a reference.

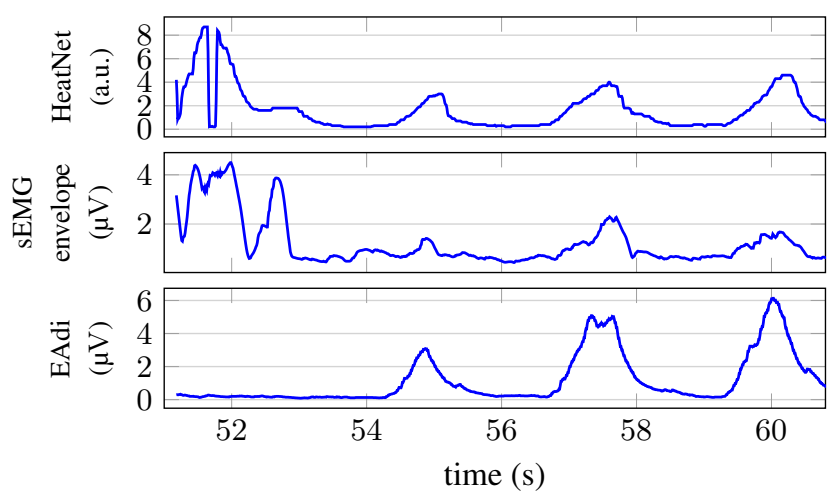

Fig. 3: An instance of failure for the neural network predictor at $t=52 \mathrm{~s}$ : Both the envelope and the HeatNet signal contain large artifacts, which can probably be prescribed to muscle crosstalk.

\section{Conclusion}

In this paper, different architectures have been evaluated for the task of predicting the internal diaphragm activity from multiple surface EMG signals. The heatmap-based regression approach outperformed the direct regression architecture, with a PCC of $0.830 \pm 0.193$, a MAE of $0.835 \pm 0.700$ and a HS of $0.888 \pm 0.044$ compared to EAdi. The prediction of diaphragm activity was substantially improved compared to the conventional single-channel sEMG envelope. Based on the learned relationship between the externally measured sEMG signals and the internal diaphragm acitvity measured by a nasogastric catheter we hypothesize, that the former may be used as a surrogate for EAdi in clinical applications. The results further confirm the findings of Bellani et al. [1] that the sEMG might be a viable method for noninvasively assessing respiration and neural output of mechanically ventilated patients. Furthermore, the possibility of doing inference on unseen sEMG data in real-time, even on CPU, might promote the feasibility of our approach in the clinical arena, e.g. for the detection of PVA. One of the remaining challenges is the rejection of muscle crosstalk in the sEMG, which we will further investigate in our future work.

\section{References}

[1] G. Bellani et al. Measurement of diaphragmatic electrical activity by surface electromyography in intubated subjects and its relationship with inspiratory effort. Respiratory Care, 63(11):1341-1349, 2018.

[2] L. Blanch et al. Asynchronies during mechanical ventilation are associated with mortality. Intensive Care Medicine, 41(4):633-641, 2015.

[3] W. Dai, C. Dai, S. Qu, J. Li, and S. Das. Very deep convolutional neural networks for raw waveforms. In 2017 IEEE International Conference on Acoustics, Speech and Signal Processing (ICASSP), pages 421-425. IEEE, 2017.

[4] L. Estrada, A. Torres, L. Sarlabous, and R. Jané. Onset and offset estimation of the neural inspiratory time in surface diaphragm electromyography: A pilot study in healthy subjects. IEEE Journal of Biomedical and Health Informatics, 22(1):67-76, 2018.

[5] G. Grasselli, M. Pozzi, and G. Bellani. Monitoring Respiratory Effort by Means of the Electrical Activity of the Diaphragm, pages 299-310. Springer, Cham, 2016.

[6] K. He, X. Zhang, S. Ren, and J. Sun. Deep residual learning for image recognition. In Proceedings of the IEEE conference on computer vision and pattern recognition, pages $770-778$, 2016.

[7] S. loffe and C. Szegedy. Batch normalization: Accelerating deep network training by reducing internal covariate shift. arXiv preprint arXiv:1502.03167, 2015.

[8] Diederik P Kingma and Jimmy Ba. Adam: A method for stochastic optimization. arXiv preprint arXiv:1412.6980, 2014.

[9] A. Newell, K. Yang, and J. Deng. Stacked hourglass networks for human pose estimation. In European Conference on Computer Vision, pages 483-499. Springer, 2016.

[10] A. Paszke, S. Gross, S. Chintala, G. Chanan, E. Yang, Z. DeVito, Z. Lin, A. Desmaison, L. Antiga, and A. Lerer. Automatic differentiation in pytorch. 2017.

[11] C. Payer, D. Štern, H. Bischof, and M. Urschler. Regressing heatmaps for multiple landmark localization using cnns. In International Conference on Medical Image Computing and Computer-Assisted Intervention, pages 230-238. Springer, 2016.

[12] A. W. Thille, Pablo Rodriguez, Belen Cabello, François Lellouche, and Laurent Brochard. Patient-ventilator asynchrony during assisted mechanical ventilation. Intensive Care Medicine, 32(10):1515-1522, 2006. 